\title{
Self-adaptive Algorithm SESP based on Energy Assessment of Wireless Sensor Network Node*
}

\author{
Huang Xuhong \\ Fujian University of Technology, Fuzhou 350014, China \\ Huangxuh1@163.com
}

\begin{abstract}
The energy of the nodes of Wireless Sensor Network (WSN) is finite. For the extension of the lifespan of the nodes, we propose a self-adaptive algorithm (SESP) based on energy assessment of WSN nodes with identical hardware model and same parameters. SESP is able to choose the cluster head without sending message to learn the surplus energy of other nodes, thus reducing in-cluster communication and simplifying the calculation of energy assessment, and eventually reducing energy consumption of WSN nodes and extending their lifespan.
\end{abstract}

Index Terms - WSN, energy consumption of WSN node, energy assessment, self-adaptive algorithm

\section{Introduction}

The node energy of Wirelss Sensor Network (WSN) is finite. And WSN is out of use with the exhaustion of the node energy. Many studies have shown that the wireless transmission consumes the largest proportion of the whole node energy[1][2], with the consumption of transmitting a bit of datum 800 times more than that of executing a command[1]. Therefore, minimizing the transmission energy consumption is the key to reduce the node energy consumption and prolong the life of WSN. Also important for the extension of the lifespan is to balance node energy consumption and make full use of the energy of each node. To tackle the issue, the paper elaborates a self-adaptive algorithm SESP based on the assessment of the residual energy of nodes.

\section{The Concumption of Node Energy and Energy-Saving Measures}

The major energy consumers of WSN node are sensor modulating circuit of data collection module, micro controller and memory of data processing module and the RF circuit of data transmission module.

There is little room left to tap the sensor modulating circuit for lessening the energy consumption as it consumes quite a little. The key then lies in reducing the energy dissipation of micro-controller and memory of data processing module and the RF circuit since the energy consumed in the process of data transmission results mainly from signal processing and offsetting transmission path attenuation.

The power dissipation of microprocessor consists of dynamic power dissipation and static one[4], with the former as the main contributor to energy saving. According to Vasanth\&Michael(2005)// item 5 of the references, the dynamic power of microprocessor is closely related to supply power voltage, physical capacitance and physical capacitance, which is formulated as follow:

$$
P_{D} \propto \alpha C V^{2} f
$$

In the formula, PD stands for dynamic power, $\mathrm{V}$ is supply power voltage, $\mathrm{C}$ physical capacitance, $\mathrm{f}$ physical capacitance and $\alpha$ active factor.

Therefore, reduction of dynamic power dissipation can be realized by lowering the clock frequency and supply power voltage. Vasanth\&Michael(2005) has demonstrated that lowering both the supply power voltage and clock frequency can result in the reduction of dynamic power dissipation. And ch anging the working conditions of the microprocessor from $200 \mathrm{MHz}$ and $1.5 \mathrm{~V}$ to $150 \mathrm{MHz}$ and $1.2 \mathrm{~V}$ could reduce up to $52 \%$ of the dissipation.

The management of dynamic power dissipation could also be realized by adopting Dynamic Voltage Supply(DVS) technology. DVS will dynamically adjust the working voltage and frequency of the microprocessor with the change of the node's working load, thus reduce the unnecessary power output in the comparatively free moments.

The RF circuit, among the components of the node, consumes the largest proportion of the enery. In accordance with the technological requirements of WSN node, RF circuit usually is made from popularly utilitized parts of low energy consumption, low price and with small size.

In a WSN with nodes of $\mathrm{N}$ number, apart from reducing the power dissipation of the hardware of each node, energy saving can also be achieved by reducing the workload of nodes through the energy management of in-between nodes communication.

Suppose the set of nodes is $\mathrm{U}$, and $\mathrm{U}=\left\{\mathrm{u}_{i}=\left(x_{i,} y_{i}\right), i=1,2, \ldots, n\right\}$, with $\left(x_{i}, y_{i}\right)$ indicating the location of a node. With the completion of the distribution of a WSN of general purpose, its nodes locations are fixed.

We here adjust the energy model proposed by item 2 of the references. Given that the needed energy for transmitting a unit of data from node $\mathrm{i}$ to node $\mathrm{j}$ is $E_{i j s}$, the energy for signal processing $E_{\text {proc }}$, and the energy for offsetting transmission path attenuation $E_{\text {was }}$, we have

* This work is partially supported by Natural Science Foundation of Fujian \#2008J0178 to Huang Xuhong 


$$
E_{i j s}=E_{p r o c}+E_{\text {was }}
$$

$E_{\text {proc }}$ here is relevant to the energy for encoding, modulation and wave filtering, and $E_{\text {was }}$, to the distance between node $i$ and node $j$. And if we designate the distance as $d_{i j}$, then we have the formula

$$
E_{\text {was }} \propto d i j
$$

Node $\mathrm{j}$ also needs energy to receive information. Suppose the energy for node $\mathrm{j}$ to receive a unit of data is $E_{j r} . E_{j r}$ then is relevant to the energy consumed by decoding, demodulation and wave filtering.

As the nodes locations are fixated when completing the distribution of a WSN of general purpose, the value of $d_{i j}$ becomes fixed. And when the node hardware of WSN is determined, $E_{\text {proc }}$ and $E_{j r}$ remain almost unchanged too. Accordingly, reducing the volume of transmitted data is the key for saving energy.

The major tasks done by WSN are collecting data from sensors and transmitting them to base station. And because its transmitting power is rather low, a WSN node has to communicate directly with its neighboring nodes. To ensure that those comparatively distant nodes could transmit data to base station, the nodes could be distributed in the form of a multiple hops self-organizing network. In the self-organizing network, clustering networking algorithm is capable of effectively reducing the volume of data transmission[6]. Every cluster elects a node as its own head which then collects data transmitted from other nodes and merges them. However, the cluster head would consume more energy than other nodes. In order to balance the energy consumption and prolong the life of the network, every node in a cluster should be chosen to serve as the head in turn periodically. As such, assessing the node energey consumption and electing a suitable one as the head would be of great help to extend the lifespan the network.

\section{Energy Assessment of Neighboring Nodes}

Within any of the clusters of WSN, if the neighboring nodes' residule energy could be learnt, the node with much residule energy could be chosen as new cluster head so as to balance energy consumption and maximize the lifespan of each cluster.

Different nodes in a WSN typically are made from the same model of hardware or parts. Therefore, the energy $E_{\text {proc }}$ needed by different nodes to transmit a unit of data is approximately equivalent. So is the energy $E_{j r}$ needed for receiving a unit of data. With the completion of the distribution of WSN of general purpose, $d_{i j}$ is fixated, and $E_{\text {was }}^{i j}$ (from node i to node j) and $E_{\text {was }}^{j i}$ (from node $\mathrm{j}$ to node $\mathrm{ij}$ ) are approximately identical.
Given that node $\mathrm{t}$ is the old cluster head, node $\mathrm{i}$ is one of the other nodes in the same cluster, $E_{\text {tis }}$ represents the needed energy for transmitting a unit of data from node $t$ to node $i$, $E_{\text {proc }}$ the energy needed for signal processing, $E_{\text {was }}^{s i}$ the energy offsetting the transmission parth attenuation, according to (1.2), we have the formula

$$
E_{\text {tis }}=E_{\text {proc }}+E_{\text {was }}^{s i}
$$

Let $E_{i t s}$ stand for the energy needed for transmitting a unit of data from node $\mathrm{i}$ to node $\mathrm{t}$, and $E^{i t}$ was for the energy offsetting the transmission parth attenuation, we can infer

$$
E_{i t s}=E_{p r o c}+E_{\text {was }}^{i t}
$$

Because

$$
E^{t i}{ }_{\text {was }}=E^{i t}{ }_{\text {was }}
$$

Substitute formula (2.3) for its counterpart in formula (2.2), and then compare the resulted with (2.1), we obtain

$$
E_{t i s}=E_{i t s}
$$

That is, the energy demanded for transmitting a unit of data from node $i$ to node $t$ is equivalent to that of from node $t$ to node $\mathrm{i}$.

So is the energy $E_{t r}$ needed for node $\mathrm{t}$ to receive a unit of data and $E_{i r}$ for node i to receive a unit of data.

If in a time cycle node $i$ receives $m$ units of data and dispatches $\mathrm{n}$ units of data, then the total energy consumption of node i $e_{i}(k)$ is

$$
e_{i}(k)=m E_{t r}+n E_{t i s}
$$

In other words, so long as the data volume transmitted and received by node $\mathrm{i}$ with its neighboring nodes in a time cycle can be obtained, the total energy consumption of node $i$ in the time cycle can be calculated and thus its residule energy can be figured out.

With new cluster head being chosen, the old heads will transmit with minimal transmitting power within them the messages from the new head. And the new head, with its own confirmation of the identity, will broadcast among its neighboring heads and make them know its identity message with rather big transmitting power.

\section{SESP Self-Adaptive In-Cluster Route Algorithm}

SESP assumes that every node has different scales of transmission power. The lowest scale is used for in-cluster transmission, with a transmission radius of $R_{1}$. All nodes of the cluster are located within the circular area with a radius of $R_{1} / 2$, which means all cluster nodes can communicate directly 
with each other. The higher scales of transimission power are used for inter-cluster transmission, with a transimission radius of $R_{2}$.

If the network lifespan is divided into several life cycles based on a fixed time step length, and the length of a life cycle is $l$, then the time step length is $1 / l$. And the whole network life cycle $\mathrm{L}$ is

$$
L=N l
$$

Before the end of every cycle, the current cluster head assesses each node's residule energy with the above-mentioned SESP, and chooses the next head for head shift.

Apart from the head, other nodes within the cycle (suppose it is the k-th one) communicate only with the head, thus forming a head-centered star-network structure as indicated in figure 1 .

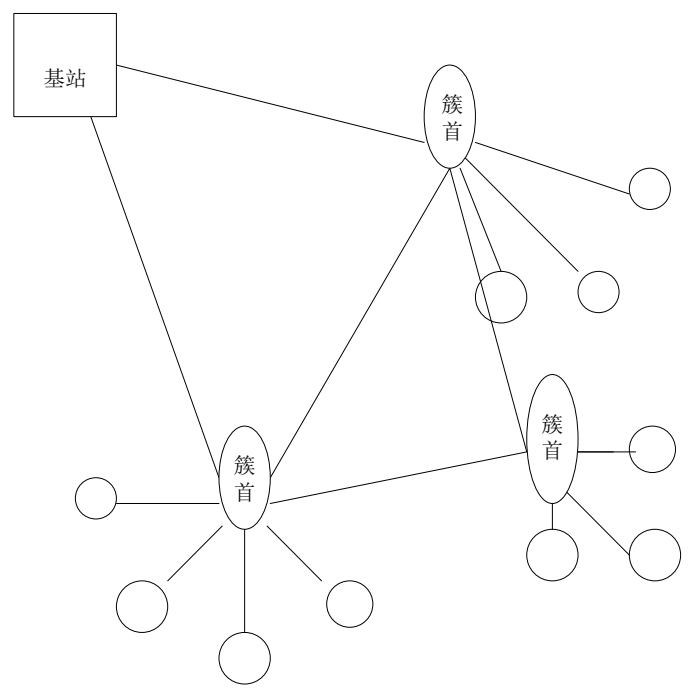

Fig. 1 Network structure

Then we can calculate the data volume $\mathrm{n}$ received and the data volume $\mathrm{m}$ transmitted by node $\mathrm{i}$ in the cluster. At the end of the $\mathrm{k}$-th cycle, the data volume $\mathrm{n}$ received and the data volume $\mathrm{m}$ dispatched by the head are respectively equivalent to that transmitted and that received by node $\mathrm{i}$. Substituting $\mathrm{m}$ and $\mathrm{n}$ for their counterparts in the formula (2.4), the energy consumption $e_{i}(k)$ in the cycle of node i can be obtained. Combined with the energy consumed in the previous cycles, the total energy consumption at present of node $\mathrm{i} E_{i}(k)$ is

$$
E_{i}(k)=e_{i}(k)+\sum_{r=1}^{k-1} e_{i}(r)
$$

Let the initial energy of each node be $E_{i}^{0}$, and the present residule energy of node i be $E_{i}^{r}(k)$, then

$$
E_{i}^{r}(k)=E_{i}^{0}-E_{i}(k)
$$

Choose the node with maximal residule energy $E_{i}^{r}(k)$ as the next cluster head.

Every node saves a message of its energy consumption in the cluster. Meanwhile, the head builds a list of total energy consumption, recording each in-cluster node's $\sum_{r=1}^{k-1} e_{i}(r)$, the head's own message included, and the locations of the nodes. In the head shift, the list will be transferred to the new head. At the end of next cycle, the new head can use the list to assess the energy consumption of the other nodes and choose the subsequent head. Such a mode will be repeated till the end of the life of the network.

\section{Conclusion}

The paper proposes a self-adaptive algorithm SESP based on energy assessment in a network and with fixed parameters. By the algorithm, no communication is needed to obtain the residule energy of other nodes when choosing cluster head, thus reducing both the in-cluster communication volume and the complexity of energy assessment calculation, and eventually saving the node energy and effectively extend the lifespan of the network.

\section{Acknowledgment}

This research is partially supported by Natural Science Foundation of Fujian Province Of China (2008J0178).

\section{References}

[1] Samuel Madden, Michael J. Franklin, Joseph M. Hellerstein, Wei Hong. Tag: a tiny aggregation service for ad-hoc sensor networks. Proc. of OSDI, 2002.

[2] W. Heinzelman, A. Chandrakasan, H. Balakrishnan. An Application-Specific Protocol Architecture for Wireless Sensor Networks. IEEE Trans. Wireless Comm., Oct.2002, 1(4), pp. 660-670.

[3] S Amit, C Anantha. Dynamic Power Managementin Wireless Sensor Networks. Selected \& test of computer, 2001, 18(2): 62-74.

[4] Ju Zhipei, Hua Yanqi. Wireless sensor network node hardware modular design. Journal of University of Shanghai For Science and Technilogy, 2009, (6):20-22.

[5] V Vasanth, F Michael. Power reduction techniques for micropro cessor system. ACM computing survey, 2005, 37(3): 195-237.

[6] A. D. Amis, R. Prakash. Load Balancing Clusters in Wireless Ad Hoc Networks. Proceedings of ASSET, March 2000, pp. 25-32. 